\title{
Strong wakefields generated by a train of femtosecond bunches in a planar dielectric microstructure
}

\author{
Changbiao Wang and J. L. Hirshfield* \\ Physics Department, Yale University, New Haven, Connecticut, USA 06520 \\ and Omega-P, Inc., 199 Whitney Avenue, New Haven, Connecticut 06511, USA \\ J.-M. Fang and Thomas C. Marshall \\ Applied Physics Department, Columbia University, New York, New York 10027, USA
}

(Received 13 November 2003; published 27 May 2004)

\begin{abstract}
A tall, dielectric-lined rectangular wakefield microstructure is analyzed as a possible element of an advanced linear wakefield accelerator. This accelerator would be driven by a train of fs electron microbunches that would be chopped out of a longer bunch using a powerful $\mathrm{CO}_{2}$ laser and then formed into a train of rectangular-profile bunches using a quadrupole. The bunches set up a periodic wakefield in the microstructure that can be built up to $400-600 \mathrm{MV} / \mathrm{m}$, for example, using a train of ten 3-fs 1-pC bunches. Two major issues are examined. First, interference is studied using the particle-in-cell code KARAT between transition radiation and Cerenkov wakefield radiation, both set up by the passage of a charge bunch through a dielectric structure of finite length. Of significance is the difference in propagation speeds of transition radiation and Cerenkov radiation (which travels almost at the vacuum light speed $c$ ) and the magnitude of the respective fields. Second, stability is examined for drive and accelerated bunches using computations of test particle orbits in the longitudinal and transverse wakefields excited by the drive bunches. It is found that nearly all test electrons in the drive bunches are confined within the structure for a travel distance of $30 \mathrm{~cm}$ or more, while test electrons located in an accelerated bunch can have stable motion over greater than $30 \mathrm{~cm}$ without passing through the structure walls.
\end{abstract}

DOI: 10.1103/PhysRevSTAB.7.051301

\section{INTRODUCTION}

Acceleration of electrons in wakefields set up by a series of drive bunches in a dielectric structure has shown promise as the basis for a linear accelerator in which large acceleration gradients can be achieved [1,2]. This concept is attractive since it would not require rf power to be injected into the structure from an external source, but rather would use wakefields set up by injected bunches obtained from a conventional low energy rf linac. With the objective of realizing such an accelerator, we have recently studied wakefield generation and beam stability in tall, planar dielectric wakefield structures having micron-scale dimensions [3]. Such structures are capable of precision fabrication using microcircuit technologies and have the capability of achieving very high field gradients: indeed, analysis has recently shown that a series of ten, 3-fs 1-pC charge bunches can set up an accelerating wakefield of $\sim 500 \mathrm{MV} / \mathrm{m}$ in a structure $18.8 \mu \mathrm{m} \times 150 \mu \mathrm{m}$ in cross section [4]. The bunches in that example are $10 \mu \mathrm{m}$ wide, and dielectric slabs a few $\mu \mathrm{m}$ thick line the structure. Planar dielectric structures offer the attraction of improving the stability of the bunch motion and increasing the amount of bunch charge [5], as compared to a cylindrical structure of comparable size. It

\footnotetext{
*Corresponding author.

Email address: jay.hirshfield@yale.edu
}

PACS numbers: 41.75.Jv, 41.75.Lx, 41.75.Ht, 96.50.Pw

is the small transverse dimension of the structure that permits large wakefields to be generated.

The bunches could be obtained initially from a conventional (e.g., $500 \mathrm{MeV}$ ) rf linac and processed using a LACARA chopper [3], or possibly an IFEL [6] used as a prebuncher, so as to obtain a sequence of bunches, each a few fs in duration. A TW-level $\mathrm{CO}_{2}$ laser would be used as a modulator [3] of the original $\mathrm{ps,} \mathrm{nC}$ bunch provided by the linac to form such a sequence of short bunches, each having charge in the $\mathrm{pC}$ range. These drive bunches, which can in principle be recycled, would in practice be followed by an accelerated bunch that is situated in the accelerating phase of axial electric field $E_{z}$ which trails the drive bunch train. In this way accelerating fields comparable with those achieved in laser plasma wakefield accelerators can be generated, yet the energy is obtained largely from the rf linac source rather than from a laser. We have found that it is possible to distort the original circular cross section of the drive bunches into a nearrectangular profile by shaping the beam stop and using a quadrupole lens following LACARA, and that the nearrectangular profile can be maintained for some distance of travel without significant deterioration due to finite beam emittance [4].

In practice, a dielectric-lined waveguide has finite length, and transition radiation will be generated when a charge bunch enters the waveguide. Interference between this transition radiation and Cerenkov wakefield 
radiation was confirmed in a previous calculation [4]. But how in detail this interference affects the form of wakefields needs careful examination. Accordingly, the 3D particle-in-cell (PIC) code KARAT [7] has been used to reveal the evolution of Cerenkov radiation and transition radiation in a rectangular dielectric-lined waveguide. Three zones (transition radiation, interference, and Cerenkov radiation) are clearly identified. The Cerenkov radiation zone expands as the charge bunch advances, since, in general, transition radiation propagates more slowly than Cerenkov radiation.

Even though the planar structure analyzed in this paper is tall, it is not infinitely tall. Thus bunch stability differs from that in an idealized 2D structure where, in the limit of high beam energy, it has been shown than transverse wake forces can vanish [5]. Indeed, analysis shows that transverse wake forces decrease, as compared to longitudinal forces, as the structure height increases. However, for the range of parameters studied, transverse wake forces are not negligible and may cause the bunches to defocus and pass through the walls of a narrow structure. An estimate has been made of how far a drive bunch might travel under the influence of the transverse fields set up by the bunch without additional focusing [8], although this estimate needs to be refined by dynamical calculations. Theory developed by Park et al. [8] is employed here to investigate bunch stability using dynamical equations. In the structure under study, the wakefields are dominated by a few very closely spaced modes having wavelengths of approximately $21.2 \mu \mathrm{m}$. Our findings show that adequate stability of the drive bunches can be obtained by choosing a tall structure $(300 \mu \mathrm{m})$, and that a certain group of test particles situated initially in a grid of loci within an accelerated bunch, as depicted in Fig. 1, can be accelerated for a distance of $1 \mathrm{~m}$ without external

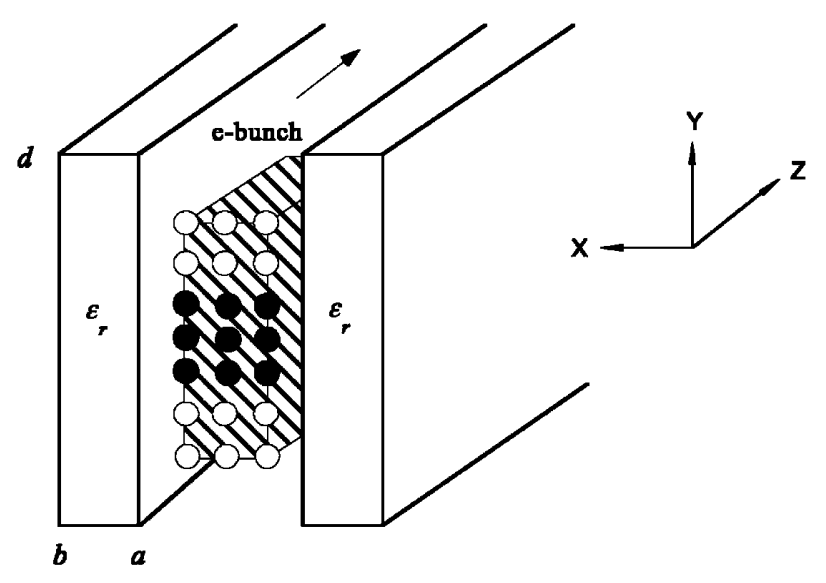

FIG. 1. Schematic slab bunch within a planar wakefield structure with a height of $2 d$, a channel width of $2 a$, and a dielectric slab thickness of $(b-a)$. All circles stand for test particles in the (decelerated) drive bunch while only filled circles stand for test particles in the accelerated bunch. focusing, maintaining a nearly stable profile and gaining an energy exceeding $550 \mathrm{MeV}$. This large magnitude of stable acceleration gradient motivates the analysis in this paper, so as to work towards realization of an advanced high-gradient linear accelerator based on the principles enunciated here.

\section{TRANSITION RADIATION EFFECTS}

The transition radiation that is emitted when the bunch enters or leaves the structure is typically omitted in analytic theoretical treatments of wakefields. The presence of transition radiation from entry and exit of a dielectric-lined structure was remarked upon in previous publications [4,9] and was recently treated analytically by Onishchenko et al. [10] using a simplified model. Qualitatively, transition radiation emitted by the entry of a bunch into the structure interferes with Cerenkov wakefield radiation in a certain zone. For the sake of convenience, three zones can be defined: the transition radiation zone where only the transition radiation dominates, the Cerenkov radiation zone where only the Cerenkov radiation dominates, and the interference zone where the two compete. The transition radiation zone plus the interference zone extends from the point where the bunch enters the structure to a front that expands with an effective group velocity of transition radiation waves in the structure. As the bunch and its wakefield move with nearly the speed of light, the expanding Cerenkov radiation zone between the interference zone and the bunch fills with wakefield radiation which resembles that emitted by the bunch in an infinitely long structure. But since the group velocity depends on the mode frequency, the transition radiation and interference zones would not be very distinct in cases of multimode operation (see, e.g., [9]). The respective zones are distinct in the example of wakefield structures in which only one mode is excited [10], which approximates the case studied here.

The first example of studies [4] described here of wakefields set up by a bunch entering a rectangular structure is for a structure with dimensions $2 d=150 \mu \mathrm{m}, 2 a=$ $15 \mu \mathrm{m}, 2 b=18.8 \mu \mathrm{m}$, and dielectric constant $\varepsilon_{r}=3.0$. The bunch height is also $150 \mu \mathrm{m}$, while the width is $10 \mu \mathrm{m}$ and the length is $1 \mu \mathrm{m}$; the bunch charge is $1 \mathrm{pC}$ and its energy is $500 \mathrm{MeV}$. The motion of the bunch was followed for $60 \mu \mathrm{m}$, and an axial field intensity contour plot in the $z-x$ plane is presented in Fig. 2. Following the bunch (located but not shown at the right-hand side at $z=$ $60 \mu \mathrm{m})$ is seen one complete wakefield period of $E_{z}$; to the left of that $(z<30 \mu \mathrm{m})$ the field structure is more complex. This is the zone where transition radiation appears. However, one finds that wakefield radiation by itself is quite uniform over the $x-y$ plane for this choice of parameters, as shown in Fig. 3, showing that near its center a tall rectangular structure can be approximated by 


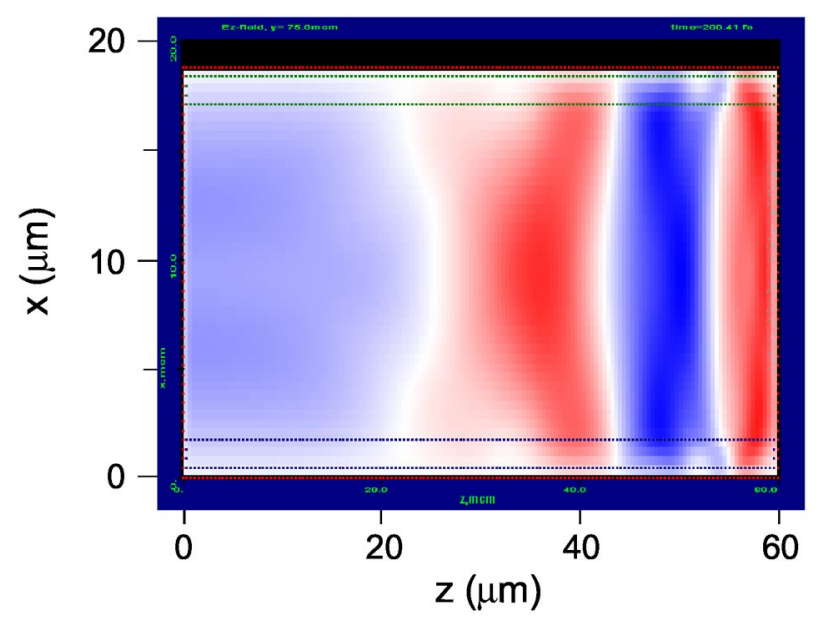

FIG. 2. (Color) Wakefield from a single bunch (located at $z=$ $60 \mu \mathrm{m}$ ), moving to the right, shown in the $z-x$ plane at a $y$-plane location halfway between the top and the bottom. The dielectric boundaries are the horizontal lines at $x=1.9$ and $16.9 \mu \mathrm{m}$. The color scale on the right is proportional to the field intensity, and the maximum is $\pm 40 \mathrm{MV} / \mathrm{m}$ in magnitude. Note the diffuse region for $z<30 \mu \mathrm{m}$ due to the interference of the wakefield and transition radiation.

its 2D counterpart. The maximum axial field from this single bunch is about $40 \mathrm{MV} / \mathrm{m}$.

In order to follow the development of the transition radiation interference pattern further using KARAT, it was necessary to reduce the bunch height to $30 \mu \mathrm{m}$ and the bunch charge proportionately, so as to work within the available computer capabilities. This choice introduces

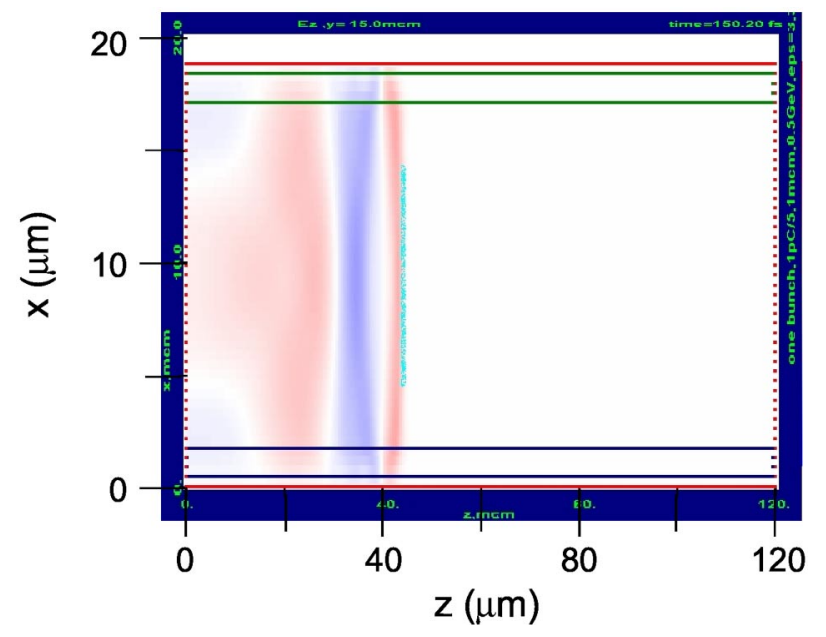

FIG. 4. (Color) Pattern of axial electric field, shown in the $z-x$ plane at midplane $y=0$ when a bunch has traveled $150 \mathrm{fs}$ from the point of entry on the left. Since the transition radiation is weaker and the Cerenkov radiation has periodicity, the three regions can be approximately distinguished: transition radiation region $(z<13 \mu \mathrm{m})$, interference region $(13<z<$ $26 \mu \mathrm{m})$, and Cerenkov radiation region $(26<z<45 \mu \mathrm{m})$. In this figure, the total axial length is $120 \mu \mathrm{m}$.

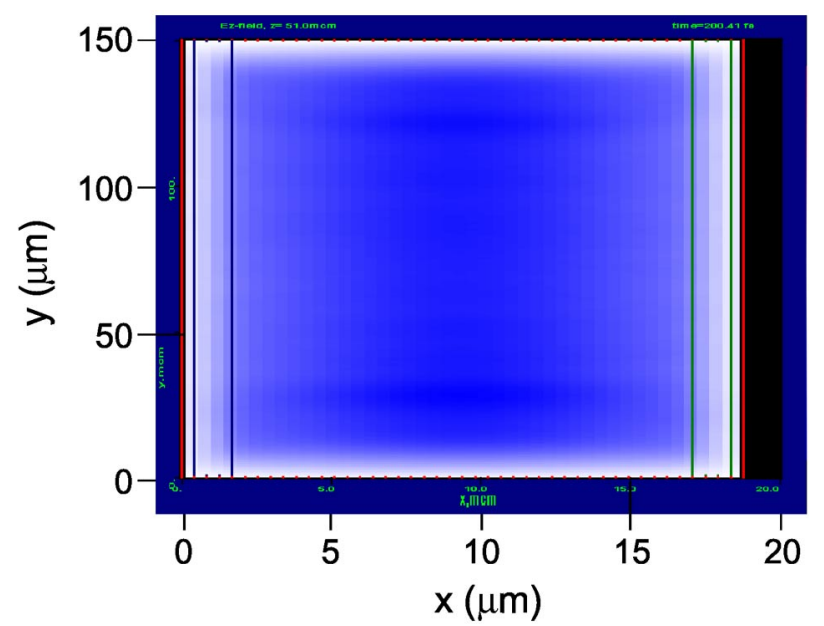

FIG. 3. (Color) Wakefield in the $x-y$ plane at $z=51 \mu \mathrm{m}$. The moving bunch is located at $z=60 \mu \mathrm{m}$. The vertical and horizontal scales are not the same: $150 \mu \mathrm{m}$ tall by $18.8 \mu \mathrm{m}$ wide. Note the field is nearly uniform except for around the two $y$ ends. The dielectric boundaries are shown as vertical lines on the left and the right.

3D features into the wakefield pattern, but if we examine the axial electric field at $y=0$, we can get a good understanding of how these fields evolve. This is shown in Figs. 4 and 5, after the bunch has traveled first 150 fs (45 $\mu \mathrm{m}$, Fig. 4) and then 350 fs (105 $\mu \mathrm{m}$, Fig. 5) from the point of entry on the left. The interference zone where transition radiation interferes with wakefield radiation moves more slowly than the wakefield radiation itself, which follows the bunch at the bunch speed $(\sim c)$. Thus as the bunch moves along the structure, the Cerenkov

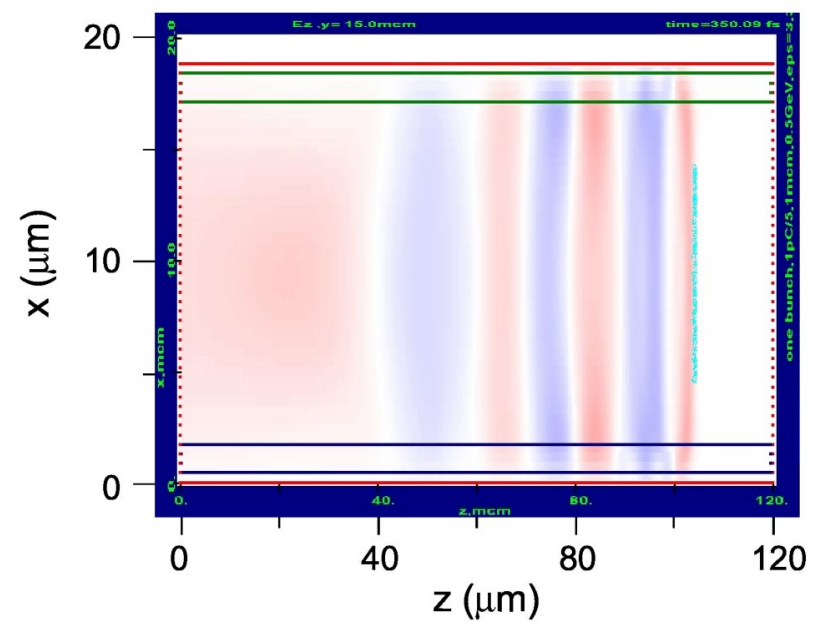

FIG. 5. (Color) Pattern when the bunch has traveled $350 \mathrm{fs}$ from the point of entry on the left. It is seen that all the transition radiation region $(z<40 \mu \mathrm{m})$, the interference region $(40<z<60 \mu \mathrm{m})$, and the Cerenkov radiation region $(60<$ $z<105 \mu \mathrm{m})$ have expanded, with the Cerenkov radiation region filled with more than two periods of wakefield radiation. 


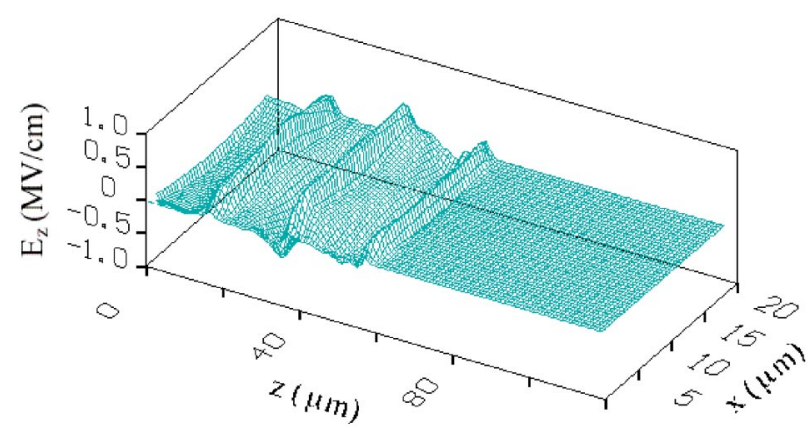

FIG. 6. (Color) Superposition of axial field $E_{z}$ excited by three individual bunches in the $z-x$ plane at the midplane $y=0$ at $200 \mathrm{fs}$ after entry of the first bunch into the structure. The third bunch still is in the transition zone and the fields of the three bunches do not add up at the third bunch position.

radiation zone between the interference zone and the bunch expands and fills with periodic $(\sim 20 \mu \mathrm{m})$ wakefield radiation, just as would be predicted by overlooking transition radiation. In this example, the boundary between the Cerenkov radiation zone and the interference zone appears to move at about $1.7 \times 10^{10} \mathrm{~cm} / \mathrm{s}$, close to $c / \varepsilon_{r}^{1 / 2}$. The magnitude of the interference radiation, about $10 \mathrm{MV} / \mathrm{m}$, is consistent with that obtained in the simplified analytic model of Onishchenko et al. [10].

A high-gradient accelerating field can be obtained by using a train of bunches, carefully spaced by the wakefield period so that constructive superposition of the wakefields of the individual bunches occurs [1]. In this case as well, transition radiation has an effect on the superposition. We demonstrate this by modeling a train of three drive bunches placed one period apart; all other parameters are the same as above. Figures 6 and 7 show the axial field $E_{z}$ in the $x-z$ plane at the midplane $y=0$ at 200 and $250 \mathrm{fs}(60$ and $75 \mu \mathrm{m})$ after entry of the first bunch into the structure. In Fig. 6 one sees the buildup of fields for the first two bunches. The third bunch is still in the transition zone and one can see that the fields of the three bunches do not add up linearly at the third bunch

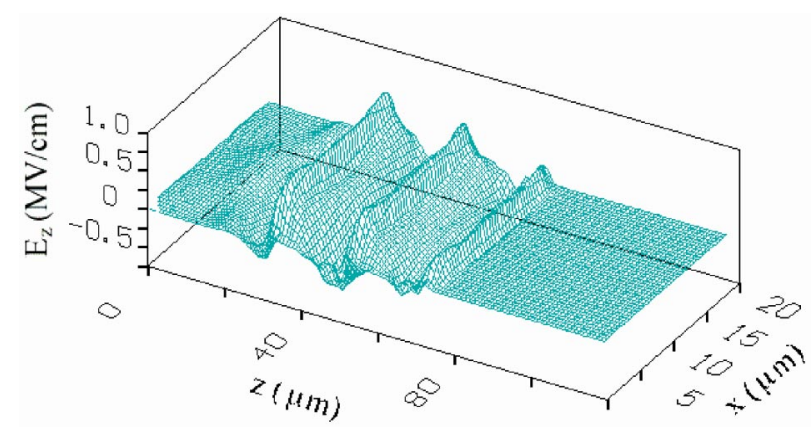

FIG. 7. (Color) Superposition of axial field $E_{z}$ excited by the three bunches at $250 \mathrm{fs}$ after entry of the first bunch into the structure. It is seen that the fields of the three bunches add at the third bunch position (see Fig. 9).

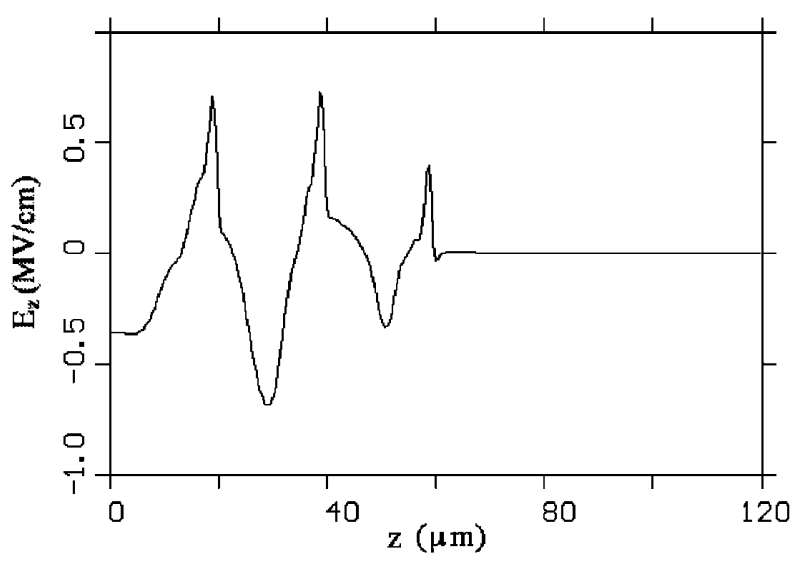

FIG. 8. Dependence of axial field $E_{z}$ on axial distance $z$ at $x=0$ and $y=0$ for Fig. 6 . It is seen that the magnitudes of the electric field at the second and third drive bunch positions are the same.

position. A more quantitative rendering of this is shown in Fig. 8. The individual magnitudes of the electric field $E_{z}$ of the second and third drive bunches are the same. As the bunches advance further (Figs. 7 and 9 at $250 \mathrm{fs}$ ), progressive buildup of the wakefields is obtained. These results suggest that transition radiation interference might well limit superposition of fields for a system that uses a very long train of drive bunches to build up the accelerating wakefield in a short structure [11].

\section{STABILITY OF PARTICLE MOTION}

A nonzero transverse gradient of $E_{z}$ must result in variation of transverse wakefields with axial distance, following the Panofsky-Wenzel theorem. So although the $E_{z}$ component can be nearly uniform in $x$ and $y$ at certain distances behind the drive bunch (see Fig. 3), bunch stability must be examined because even small transverse wakefields may cause the bunches to strike

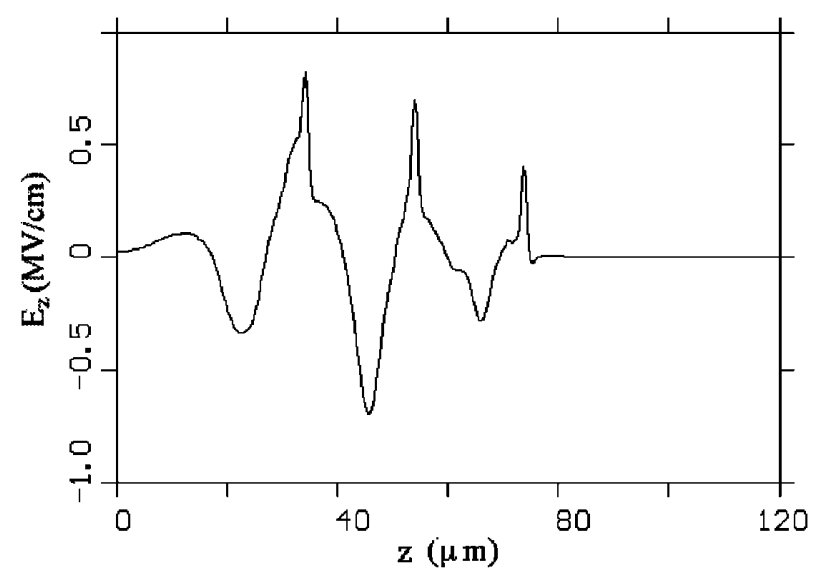

FIG. 9. Dependence of axial field $E_{z}$ on axial distance $z$ at $x=0$ and $y=0$ for Fig. 7. The electric field at the third bunch position is considerably stronger than at the second bunch. 
and penetrate the walls of this small structure as they move along an axial distance of practical length. A complete theory for excitation of wakefields in 3D planar dielectric-lined structures, similar to the theory for cylindrical devices [12], has been formulated [8]. Park has also written a code [13] based on his theory to calculate distributions of wakefield forces excited by a train of rigid electron bunches moving at constant velocity. The code is used here to calculate dynamics for test particles by adding a routine that solves the Lorentz force equations, in which the wakefield forces are given as in Ref. [8]. All test particles are initialized to have the same velocity, and the effect of beam emittance is discussed in Sec. IV.

In this computation, the dimensions of the dielectric structure shown in Fig. 1 are taken to be $2 d=300 \mu \mathrm{m}$, $2 a=15 \mu \mathrm{m}$, and $(b-a)=1.9 \mu \mathrm{m}$, and again the dielectric constant $\varepsilon_{r}$ is 3.0. For the distribution of charge within a bunch, we take a rectangular shape in the $x$ and $z$ directions, but choose a Gaussian profile for the $y$ (tall) direction (see Appendix A). The bunches have an rms height of $70.7 \mu \mathrm{m}$, a width of $10 \mu \mathrm{m}$, and a length of $1 \mu \mathrm{m}(3 \mathrm{fs})$, with each bunch containing $1 \mathrm{pC}$ of charge, and the bunch centers are set on the axis of symmetry. The initial velocity of the test particles is taken to be the same as the drive bunch, which has a relativistic factor $\gamma=$ $1000(510.5 \mathrm{MeV})$. Self-field effects and the reaction of wakefields on the drive bunches are neglected. In the simulations, $11 \times 81=891$ computational particles situated initially in an $x-y$ grid of loci were used to calculate the interaction between wakefields and test particles. The test particles in the drive bunch are initially distributed within a $10 \mu \mathrm{m} \times 159.2 \mu \mathrm{m}$ rectangle, while the test particles in the accelerated bunch are located within a $10 \mu \mathrm{m} \times 31.8 \mu \mathrm{m}$ rectangle.

The phase of injection of the accelerated bunch can be optimized. At a distance behind the last drive bunch appropriate for acceleration, the transverse wakefields are small while the axial field is large. Since the axial slippage between the wakefields and accelerated particles is negligible, one can estimate which wakefield peaks are potentially favorable for acceleration using the initial field distribution. Therefore, we first find the possible positions and then do dynamic simulations one by one to obtain the optimum injection location from the viewpoint of stability.

We begin by showing in Fig. 10 the amplitude spectrum of the modes excited by a single drive bunch. We observe that the spectrum is dominated by a few modes, having mode indices $n_{x}=1$ and $n_{y}=1,3$, and 5, corresponding to wavelengths of $21.75,21.56$, and $21.19 \mu \mathrm{m}$. If the distribution of charge within the bunch were uniform, a broader spectrum of $y$ eigenmodes would be excited, leading to the unfavorable effect of loss of bunch charge during the motion. Thus the optimum choice of the distribution and its height is that which retains the most

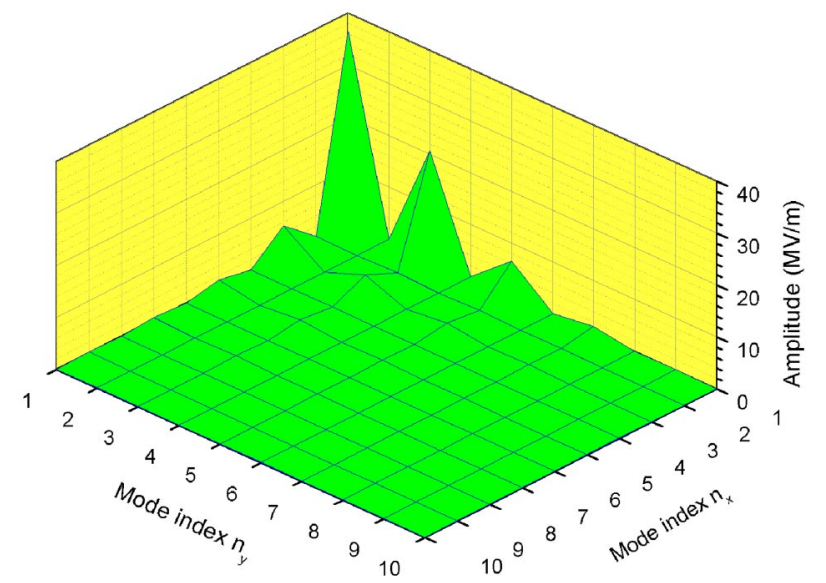

FIG. 10. (Color) Mode amplitude vs mode indices $n_{x}$ and $n_{y}$ for one drive bunch. It is seen that the modes with $\left(n_{x}=1, n_{y}=1\right.$, $3,5)$ corresponding to the wavelengths $21.75,21.56$, and $21.19 \mu \mathrm{m}$ are dominant $(36.6,20.6$, and $6.6 \mathrm{MV} / \mathrm{m})$, and next the modes with $\left(n_{x}=3, n_{y}=1,3,5\right)$ corresponding to the wavelengths $5.02,5.01$, and $5.00 \mu \mathrm{m}$ are relatively weak $(5.3,3.1$, and $1.0 \mathrm{MV} / \mathrm{m})$. The modes with even $n_{y}$ cannot be excited and those modes with $n_{y}>10$ and $n_{x}>5$ can be well ignored in computation.

charge as the drive bunches move along the device. The spectrum also shows us that the modes with $n_{y}>10$ and $n_{x}>5$ (all at short wavelengths less than $2.2 \mu \mathrm{m}$ ) can be safely ignored in computation since they are relatively weak $(<0.44 \mathrm{MV} / \mathrm{m})$.

\section{A. Motion of drive bunches}

Coherent buildup of wakefields from successive drive bunches requires a fixed bunch spacing given by the wakefield period $(21.2 \mu \mathrm{m})$. For this configuration, we provide in this section evidence of bunch stability by computing orbits of particles in the last bunch in a periodic bunch train, assuming that the preceding drive bunches are rigid. Of course, this assumption cannot give a complete understanding of stability for the entire bunch train. However, it does provide guidance, in that serious signs of instability and particle loss in this simple model would surely call into question the entire concept of using a train of drive bunches to build up a strong wakefield. In the example analyzed here, the first drive bunch is set at $z=1200 \mu \mathrm{m}$ and the 10th drive bunch is at $1009.2 \mu \mathrm{m}$. The stability of the 10th drive bunch is examined by initially setting test particles at $z=$ $1009.2 \mu \mathrm{m}$, where the axial wakefield force on the axis is nearly maximum, equal to $-430.7 \mathrm{MeV} / \mathrm{cm}$, as accumulated from the first nine drive bunches.

It is helpful to take a look at the configuration of wakefield forces to understand interactions between wakefields and test particles. The wakefield forces $F_{x}$, $F_{y}$, and $F_{z}$ at $z=1009.2 \mu \mathrm{m}$ are shown in Figs. 11-13. It is seen from Fig. 11 that $F_{x}$ is antisymmetric with respect 


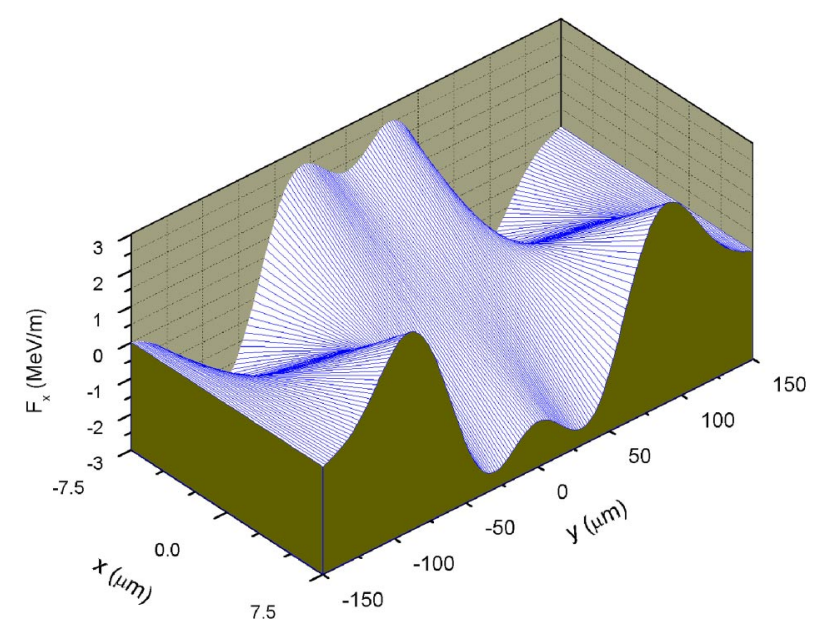

FIG. 11. (Color) Dependence of $F_{x}$ on $x$ and $y$ at $z=$ 1009.2 $\mu \mathrm{m} . F_{x}$ is focusing in the $x$ direction when $x F_{x}<0$, while $F_{x}$ is defocusing when $x F_{x}>0$. It is seen that $F_{x}$ is focusing in the region: $-60.4<y<60.4 \mu \mathrm{m}$, while defocusing in the two regions: $60.4<|y|<150 \mu \mathrm{m}$.

to $x$ and symmetric with respect to $y$. The magnitude of $F_{x}$ increases nearly linearly with $|x|$, and $F_{x}$ is either focusing or defocusing in the $x$ direction, depending on $y . F_{x}$ is focusing in the region: $-60.4<y<60.4 \mu \mathrm{m}$, while defocusing in the two regions: $60.4<|y|<$ $150 \mu \mathrm{m}$, for example. $F_{y}$ is symmetric with respect to $x$ and antisymmetric with respect to $y$; it varies slowly with $x$ while fluctuating up and down with $y$, as shown in Fig. 12. It is seen that $F_{y}<0$ when $0<y<131.6 \mu \mathrm{m}$; thus $F_{y}$ is focusing in the $y$ direction in the region of $|y|<$ $131.6 \mu \mathrm{m}$. It follows from above that both $F_{x}$ and $F_{y}$ are focusing in the common region $|x|<7.5 \mu \mathrm{m}$ and $|y|<$ $60.4 \mu \mathrm{m}$. $F_{z}$ is symmetric with respect to both $x$ and $y$, as shown in Fig. 13. It is seen that $F_{z}$ also varies slowly

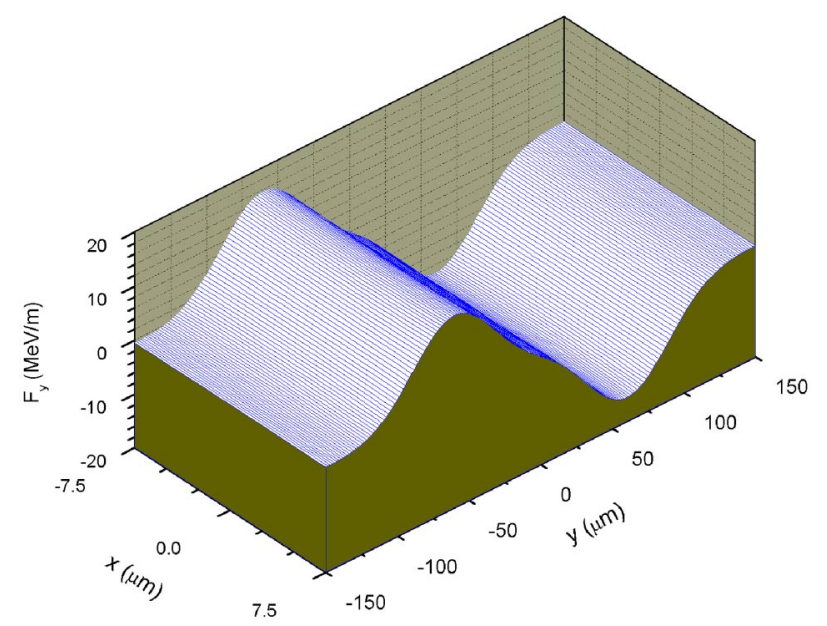

FIG. 12. (Color) Dependence of $F_{y}$ on $x$ and $y$ at $z=$ $1009.2 \mu \mathrm{m} . F_{y}$ is focusing in the $y$ direction in the region of $|y|<131.6 \mu \mathrm{m}$, leading to a common region of $|x|<7.5 \mu \mathrm{m}$ and $|y|<60.4 \mu \mathrm{m}$, where both $F_{x}$ and $F_{y}$ are focusing.

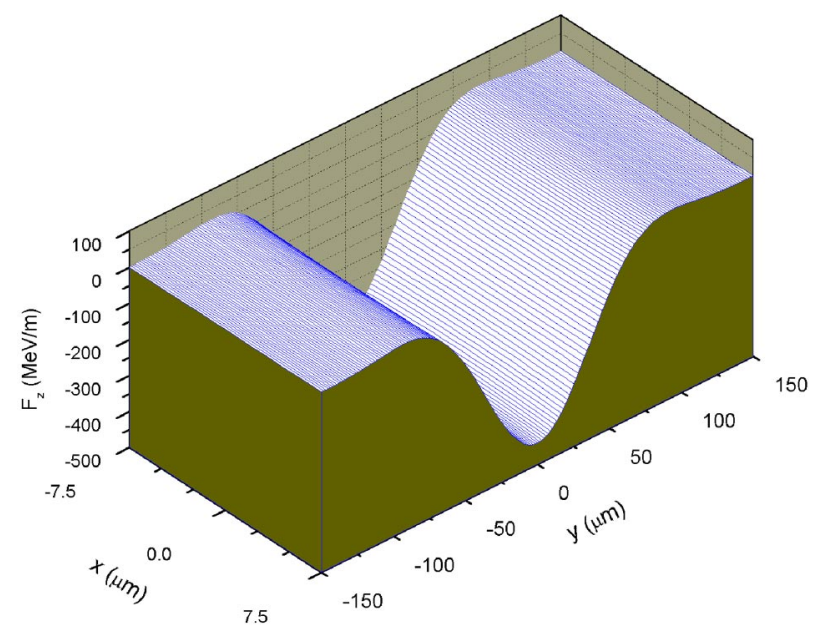

FIG. 13. (Color) Dependence of $F_{z}$ on $x$ and $y$ at $z=$ $1009.2 \mu \mathrm{m}$. It is seen that there is a small change in $F_{z}$ in the $x$ direction and a large change in the $y$ direction. $F_{z}=$ $-430.7 \mathrm{MeV} / \mathrm{m}$ at $x=y=0$.

with $x$ from $-430.7 \mathrm{MeV} / \mathrm{m}$ (at the center $x=0$ and $y=$ 0) to $-441.1 \mathrm{MeV} / \mathrm{m}$ (at the edge $x=7.5 \mu \mathrm{m}$ and $y=$ 0 ), with only a $10.4 \mathrm{MeV} / \mathrm{m}$ difference. However, it varies greatly with $y$; from $-430.7 \mathrm{MeV} / \mathrm{m}$ at the center to $16.8 \mathrm{MeV} / \mathrm{m}$ at $x=0$ and $|y|=94.5 \mu \mathrm{m}$, with about a $447 \mathrm{MeV} / \mathrm{m}$ difference, for example.

Now consider the motion of test particles of the 10th drive bunch. Figure 14 shows the distributions of test particles at $z=1009.2 \mu \mathrm{m}, 6 \mathrm{~cm}, 13.3 \mathrm{~cm}, 18 \mathrm{~cm}$, $26 \mathrm{~cm}$, and $30 \mathrm{~cm}$ under the influence of the wakefields. The test particles are initially distributed in the range extending from $x=-5 \mu \mathrm{m}$ to $+5 \mu \mathrm{m}$ and $y=$ $-79.6 \mu \mathrm{m}$ to $+79.6 \mu \mathrm{m}$, where the focusing $\left|F_{x}\right|$ takes maximum at $y= \pm 30 \mu \mathrm{m}$ and ripples as $|y|$ changes, while the focusing $F_{y}$ changes little with $x$. Both the $F_{x}$ and $F_{y}$ focusings can be easily identified from the $z=6 \mathrm{~cm}$ distribution; the $x$ dimension (width) is smallest at $y= \pm 5.5 \mu \mathrm{m}$ instead of $\pm 30 \mu \mathrm{m}$ because of $F_{y}$ focusing; the $y$ dimension (height) varies little with $x$. It is seen from Fig. 14 that the particle distribution is changed considerably during the deceleration so that some particles get lost to walls. Figure 15 shows the dependence of the average relativistic energy factor and percentage of surviving particles as a function of axial distance. All the particles go $\sim 4 \mathrm{~cm}$ without hitting any walls, and $93.7 \%$ arrive at $z=30 \mathrm{~cm}$. The particles are decelerated from $\langle\gamma\rangle=1000(510.5 \mathrm{MeV})$ to 789.7 (403.0 MeV), with 107.5 MeV lost to wakefields. The intensity of radiation generated from the ten drive bunches as they move $30 \mathrm{~cm}$ can be estimated from the energy losses to be $0.34 \mathrm{MW} / \mathrm{cm}^{2}$, which is not a level which would be destructive for the dielectric material at these wavelengths.

Simulations show that $100 \%$ of the test particles in the second drive bunch survive traveling $30 \mathrm{~cm}$, while $99.6 \%$ 

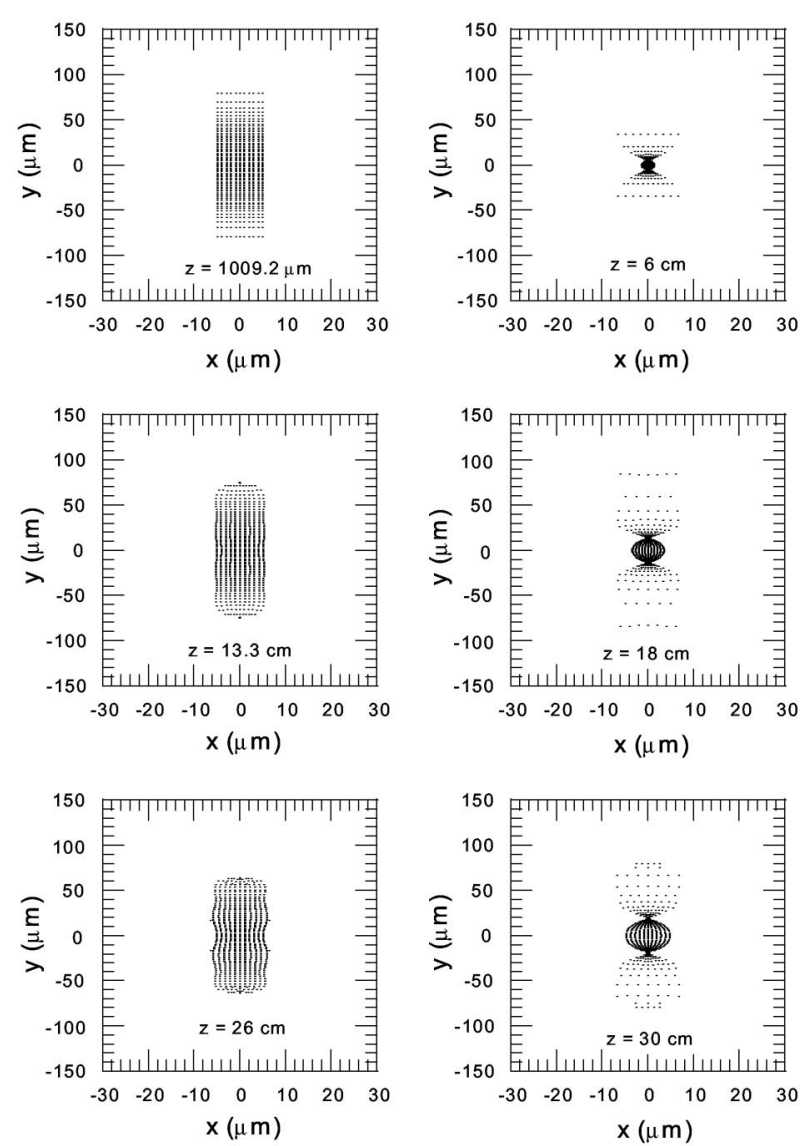

FIG. 14. Drive bunch test particle distribution at $z=$ $1009.2 \mu \mathrm{m}, 6 \mathrm{~cm}, 13.3 \mathrm{~cm}, 18 \mathrm{~cm}, 26 \mathrm{~cm}$, and $30 \mathrm{~cm}$. The test particles are initially where the tenth drive bunch would be, preceded by nine actual rigid drive bunches. The initial distribution extends from $x=-5.0$ to $5.0 \mu \mathrm{m}$ uniformly and from $y=-79.6$ to $79.6 \mu \mathrm{m}$ Gaussianly. The $F_{x}$ focusing and $F_{y}$ focusing can be easily identified from the $z=6 \mathrm{~cm}$ distribution, and the distribution experiences focusing and defocusing alternatively.

survive at the fourth and $95.5 \%$ at the seventh. The percentage reduces with increase of the bunch sequence in all nine cases. These results help to justify the assumption that prior bunches are rigid, when examining the stability of a following bunch.

One can raise this question: Might a single drive bunch provide similar test particle acceleration and stability as does a train of bunches, providing the total charge is the same? Indeed, we find that the wakefield strength generated by and the stability of the test particles following a single bunch having the same charge as the total of the original bunches are comparable to the performance of the 10-bunch train. Nevertheless, it may be easier to generate a train of high quality, low charge, short bunches rather than a single drive bunch containing the combined charge. Also, the transition radiation loss emitted by a bunch as it enters or leaves the dielectric structure module scales as the square of the bunch charge, which favors greatly the train of smaller-charge bunches.

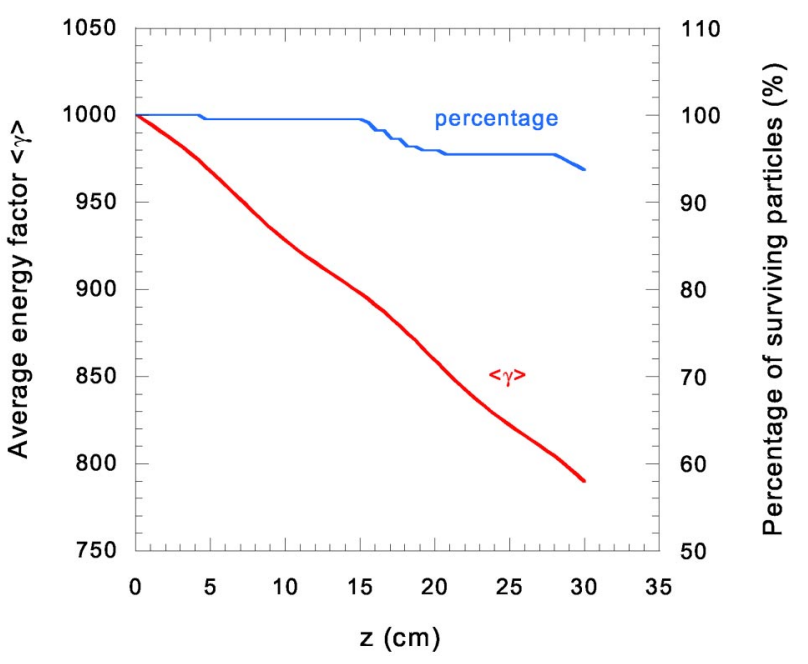

FIG. 15. (Color) Dependence of average energy factor $\langle\gamma\rangle$ and percentage of surviving particles on axial distance $z .93 .7 \%$ of the particles survive at $z=30 \mathrm{~cm}$ and they are decelerated from $\langle\gamma\rangle=1000(510.5 \mathrm{MeV})$ to $789.7(403.0 \mathrm{MeV})$, with 107.5 MeV lost to wakefields.

\section{B. Motion of an accelerated bunch}

An accelerated bunch can originate from a different source than the drive bunches, so its timing can be optimized. The stability of accelerated particles depends on positions in the wakefield and the size of the region the particles occupy. Simulations show that the test particles in an accelerated bunch following a train of ten drive bunches as described above should be situated at $z=$ $996 \mu \mathrm{m}$, where the transverse wakefield forces $F_{x}$ and $F_{y}$ are small and focusing in a rectangular region of $|x| \leq$ $5 \mu \mathrm{m}$ and $|y| \leq 15.9 \mu \mathrm{m}$, while the axial wakefield force $F_{z}$ on the axis reaches its peak, namely, $554.0 \mathrm{MeV} / \mathrm{m}$, as shown in Fig. 16.

The wakefield force configurations for $F_{x}, F_{y}$, and $F_{z}$ at $z=996 \mu \mathrm{m}$ are shown in Fig. 17. Their symmetries are the same as in Figs. 11-13. $F_{x}$ varies nearly linearly with $x$ and has zeros at $y= \pm 29.2, \pm 89.4$, and $\pm 150 \mu \mathrm{m}$, where $F_{x} \equiv 0 ; x F_{x}<0$ in the region of $|x|<7.5 \mu \mathrm{m}$ and $|y|<29.2 \mu \mathrm{m}$ and thus $F_{x}$ is focusing. $F_{y} \equiv 0$ at $y=$ 0 and it is monotone, decreasing with $y$ through zero from $y=-29.2 \mu \mathrm{m}$ to $+29.2 \mu \mathrm{m} ; y F_{y}<0$ and $F_{y}$ is also focusing in this region. $F_{z}$ increases slowly with $x$ from center to edge: $F_{z}=554.0 \mathrm{MeV} / \mathrm{m}$ at $x=0$ and $y=0$, while $F_{z}=563.6 \mathrm{MeV} / \mathrm{m}$ at $x=7.5 \mu \mathrm{m}$ and $y=0 . F_{z}$ decreases with $y$ away from the center, with $F_{z}=$ $424.5 \mathrm{MeV} / \mathrm{m}$ at $x=0$ and $y=29.2 \mu \mathrm{m}$, leading to a force difference of $139.1 \mathrm{MeV} / \mathrm{m}$ in the region mentioned above. The force difference may cause energy spread for an accelerated beam. Therefore the acceleration test particles are localized in a smaller region of $|x| \leq 5 \mu \mathrm{m}$ and $|y| \leq 15.9 \mu \mathrm{m}$ to get better particle transmission, where the force difference is reduced to about $46 \mathrm{MeV} / \mathrm{m}$. 


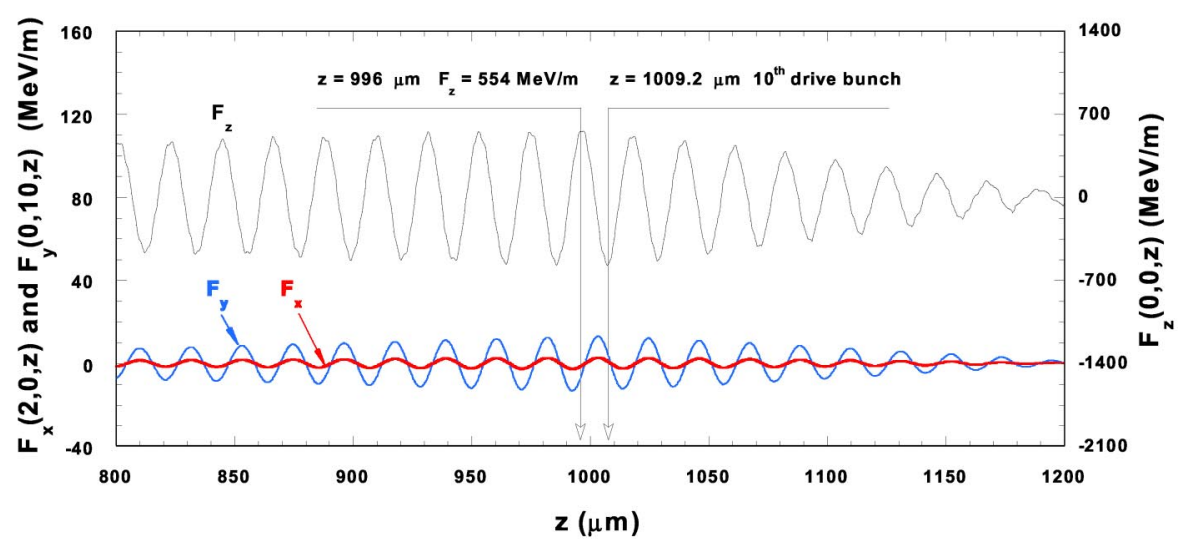

FIG. 16. (Color) Dependence of wakefield forces $F_{x}(2 \mu \mathrm{m}, 0, z), F_{y}(0,10 \mu \mathrm{m}, z)$, and $F_{z}(0,0, z)$ on axial distance $z$, excited by ten drive bunches. The stability of the accelerated bunch is examined by setting test particles at $z=996 \mu \mathrm{m} \mathrm{where} F_{x}=-1.4 \mathrm{MeV} / \mathrm{m}$ and $F_{y}=-6.6 \mathrm{MeV} / \mathrm{m}$.

Figure 18 shows 3D orbits for 15 sampled accelerated particles initially uniformly distributed within a rectangle of $|x| \leq 5 \mu \mathrm{m}$ and $|y| \leq 15 \mu \mathrm{m}$ to show how the bunch profile changes as it moves along the axis; Fig. 19 shows distributions for 891 test particles on the $x$ - $y$ planes for different axial positions. It is seen that, during the course of acceleration, the particles experience focusing and defocusing alternatively, and they are all well confined within the beam channel over a $30-\mathrm{cm}$ axial distance.

It is seen from Fig. 20 that the bunch of test particles is accelerated from $\langle\gamma\rangle=1000(510.5 \mathrm{MeV})$ to 1322.3 $(675.2 \mathrm{MeV})$ while traveling $30 \mathrm{~cm}$, corresponding to an average acceleration gradient of $549 \mathrm{MV} / \mathrm{m}$, with a final rms $\gamma$ spread of $2.9(1.5 \mathrm{MeV}$, or $0.22 \%)$. The acceleration energy increases linearly with axial distance while the energy spread goes up in a zigzag way. This behavior comes from the fact that around the focusing planes the bunch has a smaller cross section and the spread in wakefield acceleration force over the particles is also smaller, thus leading to a weaker increase of energy spread during that portion of the acceleration.

Simulations also show that about $95 \%$ of the test particles survive $100 \mathrm{~cm}$ travel; if the initial distribution area of test particles is reduced from $10 \mu \mathrm{m} \times 31.8 \mu \mathrm{m}$ to $10 \mu \mathrm{m} \times 17.5 \mu \mathrm{m}$, all the particles survive without hitting any walls and reach a final average acceleration energy of over $1 \mathrm{GeV}(\langle\gamma\rangle=2081.0)$.

\section{DISCUSSION}

This paper discussed the interference between transition radiation and Cerenkov wakefield radiation in a tall, planar dielectric microstructure, using the PIC code KARAT. This study throws light on the use of wakefields to accelerate bunches of electrons. Formerly, analysis was confined to structures of infinite length, as the initial conditions near the entry of the bunch into the structure were not treated. We have chosen conditions where mainly one wakefield mode is excited, and thus the physics is not complicated by multimode behavior. We find that because of the difference between the propagation speeds of wakefield radiation and transition radiation, a train of periodic wakefields still is excited behind the bunch as it advances into the structure. The amplitude of the transition radiation is smaller than the wakefield radiation [10], but it interferes with the latter in an expanding zone, and can affect the constructive superposition of wakefields from a train of following bunches. In general, transition radiation does not have the same periodicity as wakefield radiation, and therefore the interference can be destructive. Transition radiation also can be a problem for staged structures in a linear collider, because the radiation appears whenever a bunch enters or leaves a structure, and it is coherent in nature, scaling as the square of the bunch charge. By using many small bunches of charge to build up the wakefield, as we have done here, instead of one bunch of larger charge, the transition radiation energy loss is smaller, and thus more manageable.

We have used the motion of test particles to study the problem of stability of the driving as well as the accelerating bunches in the planar dielectric microstructure. The use of many small bunches of charge (in the $\mathrm{pC}$ range) in a structure having transverse dimensions of several $\mu \mathrm{m}$ results in very high wakefields being set up. The planar geometry also promotes exceptional stability, whereby the drive bunch train may move $\sim 30 \mathrm{~cm}$ while losing a substantial fraction of its energy but only a small fraction of its initial charge. Furthermore, a carefully positioned bunch of accelerated particles can enjoy stable high-gradient acceleration for a distance $\sim 1 \mathrm{~m}$ without the use of external focusing or guiding: a remarkable result, in view of the very small transverse dimensions of the structure. Studies of stagability would involve recycling the energy of the original drive bunches while 

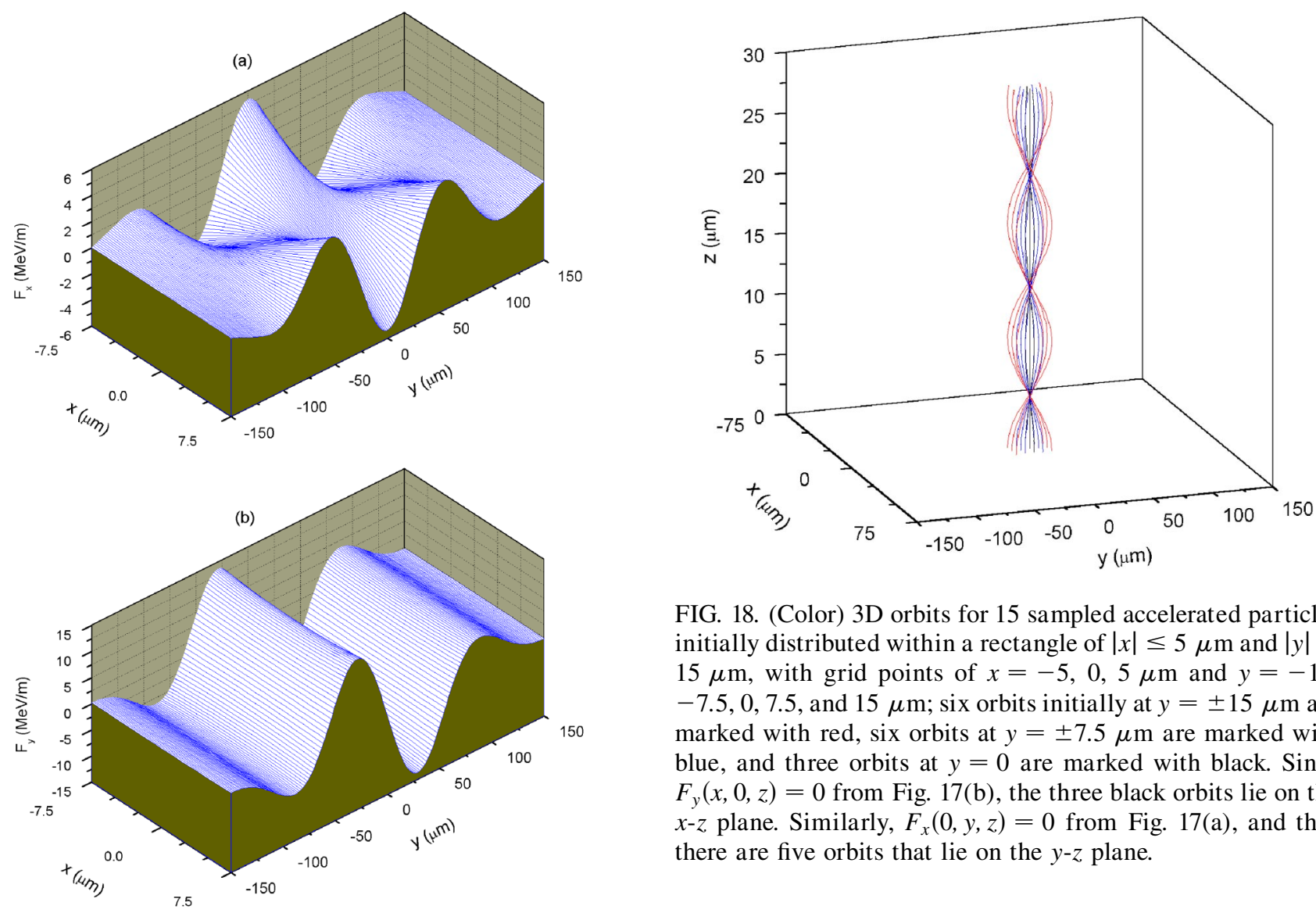

FIG. 18. (Color) 3D orbits for 15 sampled accelerated particles initially distributed within a rectangle of $|x| \leq 5 \mu \mathrm{m}$ and $|y| \leq$ $15 \mu \mathrm{m}$, with grid points of $x=-5,0,5 \mu \mathrm{m}$ and $y=-15$, $-7.5,0,7.5$, and $15 \mu \mathrm{m}$; six orbits initially at $y= \pm 15 \mu \mathrm{m}$ are marked with red, six orbits at $y= \pm 7.5 \mu \mathrm{m}$ are marked with blue, and three orbits at $y=0$ are marked with black. Since $F_{y}(x, 0, z)=0$ from Fig. 17(b), the three black orbits lie on the $x-z$ plane. Similarly, $F_{x}(0, y, z)=0$ from Fig. $17(\mathrm{a})$, and thus there are five orbits that lie on the $y-z$ plane.

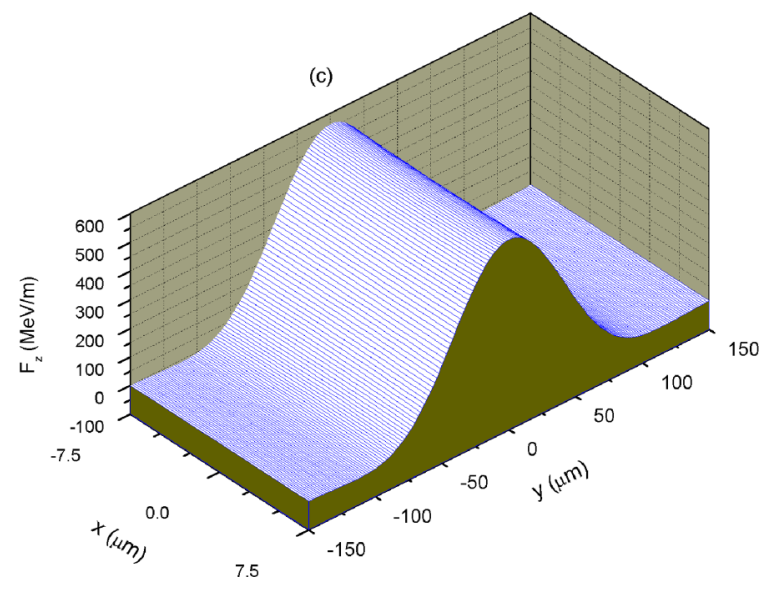

losses of charge have been shown to occur, as well as some distortion of bunch shape. However, to take these effects into account would involve a far more ambitious numerical study that is beyond the scope of this paper. Nevertheless, the remarkable stability found in our work strongly suggests that regimes of operation do exist wherein cumulative stability is found for a train of drive bunches and a following accelerated bunch.

Obtaining the results presented in this paper has required a certain amount of optimization. The choice of the bunch and structure heights, as well as the choice of the particle distribution in the bunch, was found to affect stability against transverse forces in substantial ways. For example, if the drive bunches were taken to be half the structure height with a uniform particle distribution, then acceptable travel distance without appreciable losses would be $\sim 10 \mathrm{~cm}$, which is a distance not only short of what would be an acceptable module length for a staged accelerator device, but also a distance too short to extract appreciable energy from these drive bunches. The bunch height can also exert an influence upon the stability results, as excitation of certain modes (e.g., $n_{x}=1$ and another set of drive bunches enters the next structure, followed by the original accelerated bunch; however, this problem has not been studied here.

This study of stability has used a very simplified model, in which the leading bunches do not change their shape or charge as the motion proceeds. Actually, minor $n_{y}=7$ ) was found to be harmful for particle loss. A shorter bunch will excite more modes of the structure than will a taller one, and therefore its stability may be more difficult to assure.

As seen in Sec. III, the drive bunch centers are situated on the axis of symmetry of the structure; all the modes 

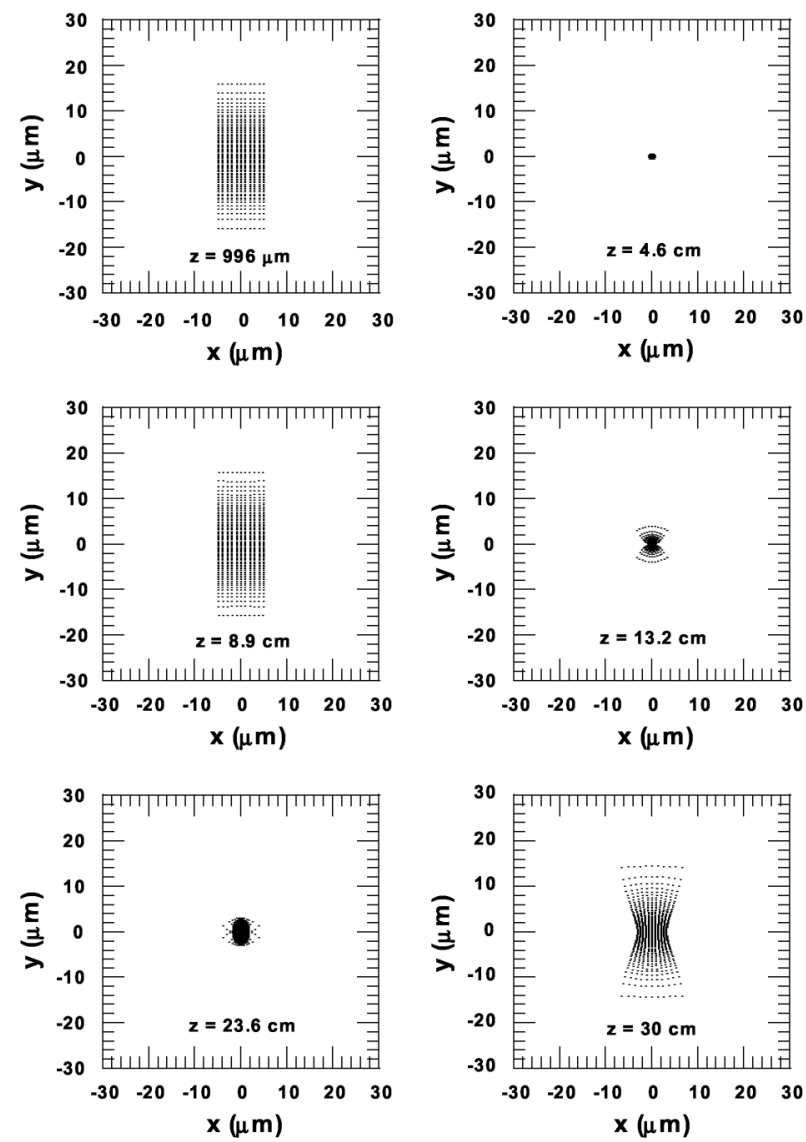

FIG. 19. Acceleration test particle distributions on the $x-y$ planes at $z=996 \mu \mathrm{m}$ (initial), $4.6 \mathrm{~cm}$ (focusing), $8.9 \mathrm{~cm}$ (defocusing), $13.2 \mathrm{~cm}$ (focusing), $23.6 \mathrm{~cm}$ (focusing), and $30 \mathrm{~cm}$ (final). 891 electrons initially distributed uniformly in the $x$ direction and Gaussianly in the $y$ direction within a rectangle of $|x| \leq 5 \mu \mathrm{m}$ and $|y| \leq 15.9 \mu \mathrm{m}$ experience focusing and defocusing alternatively, and finally all the particles reach the end of $z=30 \mathrm{~cm}$ without hitting any walls.

with even index $n_{y}$ (corresponding to those modes with $E_{z}$ antisymmetric with respect to $y$ ) cannot be excited. If the bunch centers are set off the axis, those antisymmetric modes will be encountered, as indicated in a recent study [14] where a different approach is employed to obtain wakefields, avoiding need for a direct solution of Maxwell's equations [8]. The influence of antisymmetric modes on stability is a subject for future study.

A further simplification we have used has been the choice of zero emittance electron bunches and test particles. Stability and the small transverse dimensions motivate the use of $500 \mathrm{MeV}$ bunches, which also will reduce the number of stages used in a high energy collider. Considering the smallest dimension of the system to be the $15 \mu \mathrm{m}$ channel width, one can anticipate difficulties in obtaining the propagation of bunches over a $30 \mathrm{~cm}$ distance unless the emittance is sufficiently small. We have modeled the motion of a swarm of test particles located behind the ninth drive bunch, and from this we

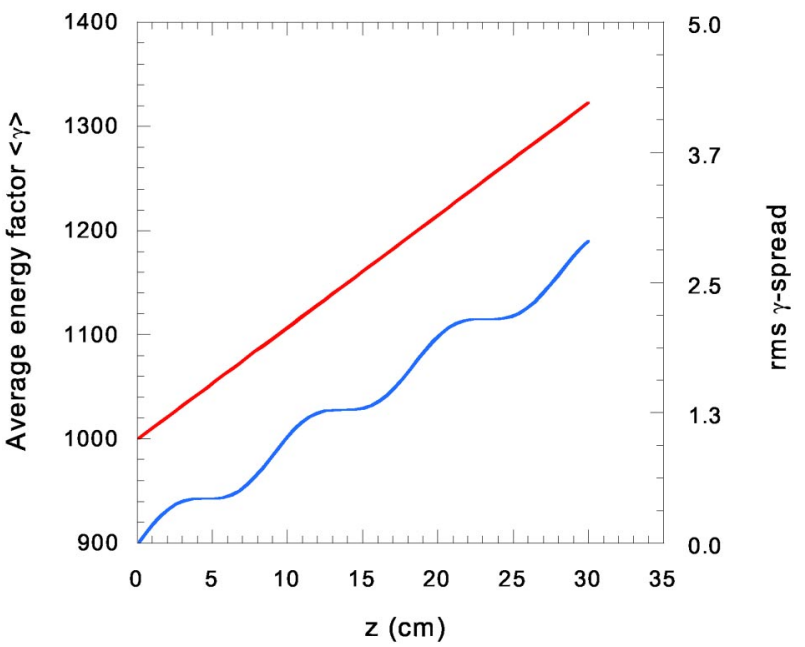

FIG. 20. (Color) Dependence of average energy factor $\langle\gamma\rangle$ and rms $\gamma$ spread on axial distance $z$. The bunch of test particles is accelerated from $\langle\gamma\rangle=1000(510.5 \mathrm{MeV})$ to $1322.3(675.2 \mathrm{MeV})$ over a 30-cm distance, with an energy gain of $164.7 \mathrm{MeV}$ and a final rms $\gamma$ spread of $2.9(1.5 \mathrm{MeV})$. It is seen that the energy spread increases much more slowly around the focusing planes $(z=4.6,13.2$, and $23.6 \mathrm{~cm})$.

estimate that a normalized emittance of $<0.5 \mathrm{~mm} \mathrm{mrad}$ would be required to obtain satisfactory transmission of the drive bunch electrons as they move in the wakefields along $30 \mathrm{~cm}$ of axial travel. This requirement, which is sensitive to the profile of $F_{x}$ in the region occupied by the test particles, is somewhat beyond the current state of the $\operatorname{art}(\sim 1 \mathrm{~mm} \mathrm{mrad})$, but it is not unreasonable to hope for improvements of this magnitude in the future. For the accelerated electrons, small finite emittance is less important because the transverse forces are stronger there and are nearly linearly focusing in the entire region occupied by these particles; we find essentially zero losses of the accelerated test particles over $100 \mathrm{~cm}$ of travel using an emittance of $0.5 \mathrm{~mm}$ mrad. Nevertheless, a more sophisticated code is needed to better understand the effects of finite emittance in this problem.

We have not yet determined what the practical features of a possible staged high energy accelerator might involve, e.g., efficiency, alignment problems, etc. However, we have studied the suitability of dielectric structures which would have a similar performance as the one described herein but which have dimensions in the millimeter range. In order to obtain the same performance, the ratio of bunch charge to aperture area $Q /(4 a d)$ must be kept the same, and the dispersion relation must yield a similar mode spectrum. As the latter requires that the dimensions must scale as the wakefield period, to construct a structure which would accept a train of bunches spaced by $9 \mathrm{~mm}$ would require a bunch charge which is $\sim 160 \mathrm{nC}$, impractically large. This shows one advantage of working with a micrometer-scale structure. 
We have chosen the wakefield period to be $21.2 \mu \mathrm{m}$ here only for purposes of illustration. In practice, a choice of $10.6 \mu \mathrm{m}$ might be advisable because the microbunches must be prepared using a laser. The dimensions of the planar dielectric device and the dielectric constant of the material would be chosen appropriately. A suitable dielectric material at $10.6 \mu \mathrm{m}$ might be $\mathrm{ZnSe}$ (refractive index $=2.40$ ), which can be formed by chemical vapor deposition [15]. This material is optically isotropic, has low losses in the far infrared, and is nearly free of dispersion.

Taken together, the results of this paper suggest that a high-gradient electron wakefield accelerator could be built with individual module lengths of $30-100 \mathrm{~cm}$, wherein stable acceleration gradients of $\sim 0.5 \mathrm{GeV} / \mathrm{m}$ could be sustained using a train of drive bunches of charge only in the $\mathrm{pC}$ range.

\section{ACKNOWLEDGMENTS}

The work was supported by the Department of Energy.

\section{APPENDIX A: INITIALIZATION OF A GAUSSIAN BEAM}

In this Appendix, the procedure used for constructing a Gaussian distribution for computations presented in this paper is described. The Gaussian distribution in the $y$ direction is assumed to be

$$
f(y)=\frac{2}{\sqrt{\pi} \Delta y_{0}} e^{-\left[\left(y-y_{0}\right) /\left(\Delta y_{0} / 2\right)\right]^{2}},
$$

where $y_{0}$ is the bunch center. The rms height for this distribution is given by $2\left(\left\langle y^{2}\right\rangle-\langle y\rangle^{2}\right)^{1 / 2}=\Delta y_{0} / \sqrt{2}=$ $70.7 \mu \mathrm{m}$ if $\Delta y_{0}=100 \mu \mathrm{m}$, for example.

Suppose that there are $N_{y}$ computational particles to be labeled in the $y$ direction. The $n$th particle location $b_{n}$ is implicitly given by the following equation:

$$
\begin{gathered}
\frac{2}{\sqrt{\pi} \Delta y_{0}} \int_{b_{n-1}}^{b_{n}} e^{-\left[\left(y^{\prime}-y_{0}\right) /\left(\Delta y_{0} / 2\right)\right]^{2}} d y^{\prime}=\frac{1}{N_{y}+1}, \\
\left(b_{0}=-\infty, n=1,2, \ldots, N_{y}\right),
\end{gathered}
$$

where the $\left(N_{y}+1\right)$ th or last particle is neglected in computations because it is represented as being at infinity.

Using the error function $\operatorname{erf}(x) \equiv(2 / \sqrt{\pi}) \int_{0}^{x} e^{-t^{2}} d t$, we obtain from Eq. (A2)

$$
\begin{gathered}
\operatorname{erf}\left(\frac{b_{n}-y_{0}}{\Delta y_{0} / 2}\right)-\operatorname{erf}\left(\frac{b_{n-1}-y_{0}}{\Delta y_{0} / 2}\right)=\frac{2}{N_{y}+1}, \\
\left(n=1,2, \ldots, N_{y}, \quad \text { and } \quad b_{0}=-\infty\right) .
\end{gathered}
$$

Equation (A3) is a recurrence equation that gives locations of each of the $N_{y}$ particles for the Gaussian distri- bution Eq. (A1). The first computational particle at $y=b_{1}$ is a proxy for all the particles ranging from $-\infty$ to $b_{1}$ in the Gaussian distribution while that at $y=+\infty$ is a corresponding proxy for those ranging from $b_{N_{y}}$ to $+\infty$. In the computations, those proxy particles are discarded, amounting to truncation of the Gaussian function. The fraction of particles truncated is $1 /\left(N_{y}+1\right)$. Obviously, accuracy in representing the Gaussian improves as $N_{y}$ increases. For example, if $N_{y}=81$, the truncated fraction is about $1.2 \%$. In this case, the truncated representation for the Gaussian function is probably sufficient for purposes of this work.

[1] T-B. Zhang, J. L. Hirshfield, T.C. Marshall, and B. Hafizi, Phys. Rev. E 56, 4647 (1997).

[2] J. G. Power, M. E. Conde, W. Gai, R. Konecny, P. Schoessow, and A. D. Kanareykin, Phys. Rev. ST Accel. Beams 3, 101302 (2000).

[3] T. C. Marshall, C. Wang, and J. L. Hirshfield, Phys. Rev. ST Accel. Beams 4, 121301 (2002).

[4] T. C. Marshall, J.-M. Fang, J. L. Hirshfield, C. Wang, V. P. Tarakanov, and S.Y. Park, in Advanced Accelerator Concepts: Tenth Workshop, edited by C. E. Clayton and P. Muggli, AIP Conf. Proc. No. 647 (AIP, New York, 2002), p. 361.

[5] A. Tremaine, J. Rosenzweig, and P. Schoessow, Phys. Rev. E 56, 7204 (1997).

[6] Y. Liu, X. J. Wang, D. B. Cline, M. Babzien, J. M. Fang, J. Gallardo, K. Kusche, I. Pogorelsky, J. Skaritka, and A. van Steenbergen, Phys. Rev. Lett. 80, 4418 (1998).

[7] V. P. Tarakanov, computer code KARAT, BRA Inc., VA, USA, 1992.

[8] S.-Y. Park, C. Wang, and J. L. Hirshfield, in Advanced Accelerator Concepts: Tenth Workshop, edited by C. E. Clayton and P. Muggli (Ref. [4]), p. 527.

[9] T. C. Marshall, J.-M. Fang, J. L. Hirshfield, and S. Y. Park, in Advanced Accelerator Concepts: Ninth Workshop, edited by P. Colestock and S. Kelley, AIP Conf. Proc. No. 569 (AIP, New York, 2001), p. 316.

[10] I. N. Onishchenko, D. Yu. Sidorenko, and G. V. Sotnikov, Phys. Rev. E 65, 066501 (2002).

[11] I. N. Onishchenko, D. Yu. Sidorenko, and G. V. Sotnikov, Ukr. J. Phys. 48, 17 (2003).

[12] S.-Y. Park and J. L. Hirshfield, Phys. Rev. E 62, 1266 (2000).

[13] S.-Y. Park (private communication).

[14] C. Jing, W. Liu, L. Xiao, W. Gai, and P. Schoessow, Phys. Rev. E 68, 016502 (2003).

[15] D. N. Nikogosyan, Properties of Optical and LaserRelated Materials: A Handbook (John Wiley \& Sons, New York, 1997), p. 286. 